\title{
Effects of Chlorthalidone and Diltiazem on Myocardial Ischemia in Elderly Patients with Hypertension and Coronary Artery Disease.
}

\author{
João Batista Serro-Azul; Rogério Silva de Paula; César Gruppi; Lígia Pinto; Humberto Pierri; \\ Amit Nussbacher; Otávio Gebara; Paulo Moffa; Antônio Carlos Pereira-Barreto; Maurício Wajngarten
}

\author{
São Paulo, SP - Brazil
}

Objective - Antihypertensive therapy with thiazides decreases coronary events in elderly patients. However, the influence of diuretics on myocardial ischemia has not been fully investigated. The aim of this study was to compare the effect of chlorthalidone and diltiazem on myocardial ischemia.

Methods - Following a randomized, double-blind, crossover protocol, we studied 15 elderly hypertensive patients aged $73.6 \pm 4.6$ years with myocardial ischemia. All patients had angiographically documented coronary artery disease. We measured patients using 48- hour ambulatory electrocardiogram monitoring and exercise testing. After a 2-week period using placebo, patients received chlorthalidone or diltiazem for 4 weeks.

Results - Both treatments lowered systolic and diastolic blood pressures. The number of ischemic episodes on ambulatory electrocardiogram recordings was reduced with the use of chlorthalidone (2.5 \pm 3.8$)$ and diltiazem

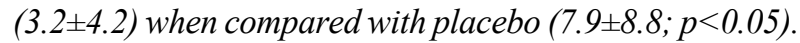
The total duration of ischemic episodes was reduced in both treatments when compared with placebo (chlorthalidone: 19.2 $\pm 31.9 \mathrm{~min}$; diltiazem: $19.3 \pm 29.6 \mathrm{~min}$; placebo: 46.1 $\pm 55.3 \min ; p<0.05$ ).

Conclusion - In elderly hypertensive patients with coronary artery disease, chlorthalidone reduced myocardial ischemia similarly to diltiazem. This result is consistent with epidemiological studies and suggests that reduction of arterial blood pressure with thiazide therapy plays an important role in decreasing myocardial ischemia.

Key words: ischemia, hypertension, diuretics, elderly.

Instituto do Coração do Hospital das Clínicas - FMUSP

Mailing address: João Batista Serro-Azul - InCor - Av. Dr. Enéas C. Aguiar, $44-$ 05403-000 - São Paulo, SP - Brazil
Hypertension is an important risk factor for coronary artery disease ${ }^{1}$, which is the major cause of death in persons aged 65 years or older ${ }^{2}$. Antihypertensive therapy with thiazide diuretics significantly reduces cardiovascular morbidity and mortality in the elderly, including coronary events ${ }^{3-5}$. Furthermore, it has been shown that diuretic therapy itself has antianginal effects and improves exercise capacity in patients with stable angina ${ }^{6}$. Despite the use of diuretics in many large trials ${ }^{3-5}$, their influence on myocardial ischemia in the elderly has not been fully investigated. The aim of this study was to compare the effect of chlorthalidone and diltiazem on myocardial ischemia in hypertensive elderly patients with coronary artery disease.

\section{Methods}

Study population - Fifteen patients (11 men, 4 women) from the geriatric outpatient clinic were selected to participate in this study approved by the Ethical Committee. Participation required signed informed consent. Patients were all over 65 years of age (73.6+4.6 years). Hypertension was diagnosed when blood pressure was above $160 / 90 \mathrm{mmHg}$ on at least 2 consecutive clinic visits. All patients had evidence of myocardial ischemia on 48-hour ambulatory electrocardiogram monitoring and on exercise treadmill testing, and angiographically documented coronary artery disease (>70\% diameter stenosis of at least 1 major coronary artery) (Table I).

None of the patients had unstable angina, previous myocardial infarction, left ventricular ejection fraction $<50 \%$, valvular disease, high degree atrioventricular block, bundle branch block, atrial flutter, or atrial fibrillation.

Study design - After a 2-week wash-out period using placebo, patients received active treatment with either chlorthalidone (25mg, once daily) or diltiazem (30mg, thrice a day) during 2 periods of 4 weeks each according to a randomized, double-blind, crossover protocol. Patients were 


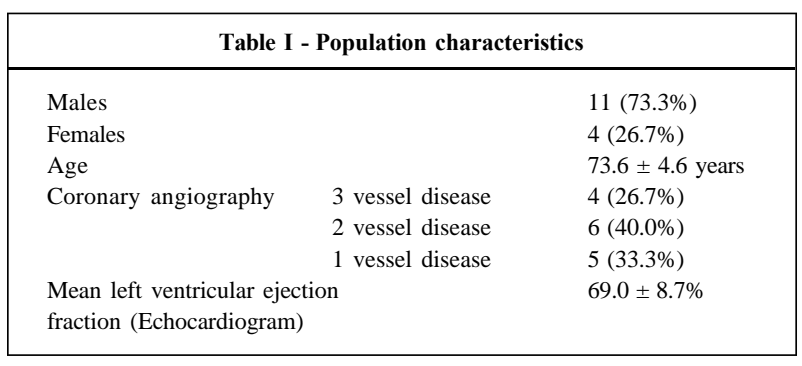

allowed to use sublingual nitrate during all periods of the study except during ambulatory electrocardiogram monitoring or 6 hours before exercise treadmill testing. After the placebo period and after each active treatment period, patients underwent a clinical evaluation, blood chemistry analysis, ambulatory electrocardiogram monitoring, and exercise testing.

Blood pressure was measured with a mercury sphygmomanometer with the patient in the supine position after 5 minutes of rest. Blood samples were obtained for the following examinations: glucose, potassium, urea, triglycerides, total cholesterol, low-density lipoprotein cholesterol (LDLcholesterol), high-density lipoprotein cholesterol (HDLcholesterol), and very-low-density lipoprotein cholesterol (VLDL-cholesterol).

Ambulatory electrocardiogram monitoring was performed over 48 hours using a magnetic tape recorder system, amplitude modulated, with a calibrated 2-channel electrocardiograms (Marquette, series 8500, Marquette Electronics Inc., Milwaukee, WI). After careful skin preparation, the recordings were accomplished with 2 bipolar leads: CM5 (channel 1) and CM1 (channel2). An ischemic episode was defined as the presence of at least 1 episode of horizontal or downsloping ST segment depression $>0.1 \mathrm{mV}$ from baseline, persisting for at least 1 minute, and separated from another episode by at least 1 minute. Holter recordings were visually analyzed on a computerized system (Laser-Holter, series 8000 T, Marquette Electronics Inc., Milwaukee, WI).

Exercise testing was performed using a computerized system (Fukuda Denshi, ML 8000, Fukuda Denshi Co. Ltd., Tokyo, Japan) with 15 leads (12 conventional leads plus CC5, CM5, and CL) according to a modified Naughton protocol. Exercise was discontinued for chest pain grade III, fatigue, dyspnea, or abnormal elevation of blood pressure. Blood pressure was measured by a sphygmomanometer (Colin, model STBP 780, Colin Medical Instruments Corp., Komaki City, Japan) at rest and at the last minute of each stage of exercise. Ischemic changes were defined as the presence of a horizontal or downsloping ST segment depression of at least $0.1 \mathrm{mV}$ from baseline at $80 \mathrm{~ms}$ after the $\mathrm{J}$ point.

Statistical analysis - Heart rate (HR), systolic blood pressure (SBP), rate-pressure product, exercise duration, and serum levels of urea, potassium, total cholesterol, LDLcholesterol, HDL-cholesterol, VLDL-cholesterol, and triglycerides were compared by repeated measures analysis of variance with multiple comparison procedures. Friedman repeated measures analysis of variance on ranks and Dunn's method were applied to variables that did not follow a normal distribution: diastolic blood pressure (DBP), time to ischemia, HR at the time of ischemia, number of ischemic episodes, total ischemia duration, numbers of ectopic atrial and ventricular beats, and glucose. Calculations were performed with the Statistical Analysis Systems; $\mathrm{p}<0.05$ was accepted as statistically significant.

\section{Results}

\section{Effects on blood pressure}

Office examination (Table II)-Both SBP and DBP were reduced by chlorthalidone and diltiazem when compared with placebo. The reduction of DBP, however, was greater with chlorthalidone than with diltiazem.

Exercise testing (Table III) - At onset of ischemia, SBP was similar in the 3 treatment groups. At peak exercise, SBP was not statistically different during treatments with chlorthalidone and diltiazem when compared with placebo.

\section{Effects on heart rate}

Office examination (Table II)-HR was not modified by either chlorthalidone or diltiazem treatments when compared with placebo.

Ambulatory electrocardiogram monitoring(Table IV) - Mean HR on 48-hour monitoring did not statistically differ during treatment with either chlorthalidone or diltiazem when compared with placebo. HR at the time of ischemic episodes also did not differ among treatments.

Exercise testing (Table III) - HR at onset of ischemia was significantly reduced with diltiazem when compared with placebo but was not modified with chlorthalidone. At peak exercise also, HR was reduced with the use of diltiazem when compared with placebo, but was not modified with the use of chlorthalidone.

\section{Effects on myocardial ischemia}

Ambulatory electrocardiogram monitoring(TableIV) - The number of ischemic episodes was reduced with the use of chlorthalidone and diltiazem when compared with placebo. Chlorthalidone, however, significantly reduced the number of ischemic episodes when compared with diltiazem. The total duration of ischemic episodes was reduced in both treatments when compared with placebo.

Exercise testing (Table III) - Exercise duration was not modified with the use of chlorthalidone when compared with placebo and diltiazem. A significant increase in exercise duration occurred with the use of diltiazem when compared with placebo. Time to onset of ischemia was not modified by either treatment when compared with placebo. At the onset of ischemia, the rate-pressure product $\left(\mathrm{HR} \times \mathrm{SBP} \times 10^{-3}\right)$ was similar in the 3 treatment groups. At peak exercise, however, no difference in the rate-pressure product among treatments occurred.

Effect on cardiac arrhythmias (Table IV) - All patients experienced cardiac arrhythmias during 48-hour ambulatory electrocardiogram monitoring. Neither chlorthalidone nor diltiazem treatments modified the number of ectopic atrial 


\begin{tabular}{|c|c|c|c|c|c|c|c|}
\hline \multicolumn{8}{|c|}{ Table II - Office data } \\
\hline & \multirow[b]{2}{*}{ Plac } & \multirow[b]{2}{*}{ Chlort } & \multirow[b]{2}{*}{ Dilt } & $\begin{array}{l}\mathrm{p} \text {-value } \\
\text { Comparison among } \\
\text { treatments }\end{array}$ & \multicolumn{2}{|c|}{ Multiple comparison procedures } & \multirow[b]{2}{*}{$\begin{array}{c}\text { Chlort } \\
\text { versus } \\
\text { Dilt }\end{array}$} \\
\hline & & & & & $\begin{array}{c}\text { Plac } \\
\text { versus } \\
\text { Chlortversus }\end{array}$ & $\begin{array}{c}\text { Plac } \\
\text { versus } \\
\text { Dilt }\end{array}$ & \\
\hline $\mathrm{HR}$ & $74.5 \pm 7.1$ & $75.2 \pm 6.4$ & $72.7 \pm 6.6$ & 0.4937 & - & - & - \\
\hline SBP & $171.3 \pm 20.0$ & $158.1 \pm 18.1$ & $160.0 \pm 20.7$ & 0.0054 & 0.0017 & 0.0303 & 0.5711 \\
\hline $\mathrm{DBP} *$ & $99.4 \pm 3.9$ & $82.7 \pm 6.2$ & $84.4 \pm 9.4$ & $<0.0001$ & $<0.05$ & $<0.05$ & $<0.05$ \\
\hline
\end{tabular}

Data are expressed as mean value \pm standard deviation * Friedman repeated measures analysis of variance on ranks Plac: placebo; Chlort: chlorthalidone; Dilt: diltiazem HR: heart rate, in beats per minute; SBP: systolic blood pressure and DBP: diastolic blood pressure, in mmHg

\begin{tabular}{|c|c|c|c|c|c|c|c|}
\hline \multicolumn{8}{|c|}{ Table III - Exercise treadmill testing data } \\
\hline & \multirow[b]{2}{*}{ Plac } & \multirow[b]{2}{*}{ Chlort } & \multirow[b]{2}{*}{ Dilt } & \multirow{2}{*}{$\begin{array}{l}\mathrm{p} \text { - value } \\
\text { Comparison among } \\
\text { treatments }\end{array}$} & \multicolumn{3}{|c|}{ Multiple comparison procedures } \\
\hline & & & & & $\begin{array}{c}\text { Plac } \\
\text { versus } \\
\text { Chlort }\end{array}$ & $\begin{array}{c}\text { Plac } \\
\text { versus } \\
\text { Dilt }\end{array}$ & $\begin{array}{c}\text { Chlort } \\
\text { versus } \\
\text { Dilt }\end{array}$ \\
\hline \multicolumn{8}{|l|}{ Onset of ischemia } \\
\hline HR & $124.3 \pm 17.5$ & $117.1 \pm 18.6$ & $113.1 \pm 13.7$ & 0.0457 & 0.0749 & 0.0128 & 0.3252 \\
\hline SBP & $187.2 \pm 27.8$ & $190.7 \pm 29.8$ & $182.7 \pm 26.1$ & 0.5937 & - & - & - \\
\hline RPP & $23.3 \pm 5.1$ & $22.6 \pm 5.6$ & $20.1 \pm 3.9$ & 0.0521 & - & - & - \\
\hline $\begin{array}{l}\text { Time to } \\
\text { ischemia * }\end{array}$ & $5.1 \pm 2.5$ & $7.2 \pm 5.2$ & $5.4 \pm 2.6$ & 0.5134 & - & - & - \\
\hline \multicolumn{8}{|l|}{ Peak of Exercise } \\
\hline $\mathrm{HR}$ & $140.3 \pm 18.2$ & $135.2 \pm 17.1$ & $133.4 \pm 15.4$ & 0.0178 & 0.0686 & 0.0041 & 0.4830 \\
\hline SBP & $191.1 \pm 34.5$ & $192.9 \pm 34.5$ & $199.5 \pm 39.5$ & 0.5391 & - & - & - \\
\hline RPP & $26.7 \pm 5.7$ & $26.3 \pm 6.9$ & $26.7 \pm 6.3$ & 0.9415 & - & - & - \\
\hline \multicolumn{8}{|l|}{ Duração do } \\
\hline Exercise duration & $11.9 \pm 7.1$ & $13.5 \pm 6.1$ & $14.6 \pm 6.0$ & 0.0244 & 0.0712 & 0.0071 & 0.1612 \\
\hline
\end{tabular}

\begin{tabular}{|c|c|c|c|c|c|c|c|}
\hline \multicolumn{8}{|c|}{ Table IV - Ambulatory electrocardiogram data } \\
\hline & \multirow[b]{2}{*}{ Plac } & \multirow[b]{2}{*}{ Chlort } & \multirow[b]{2}{*}{ Dilt } & \multirow{2}{*}{$\begin{array}{l}\mathrm{p} \text {-value } \\
\text { comparison among } \\
\text { treatments }\end{array}$} & \multicolumn{3}{|c|}{ Multiple comparison procedures } \\
\hline & & & & & $\begin{array}{c}\text { Plac } \\
\text { versus } \\
\text { Chlort }\end{array}$ & $\begin{array}{c}\text { Plac } \\
\text { versus } \\
\text { Dilt }\end{array}$ & $\begin{array}{c}\text { Chlort } \\
\text { versus } \\
\text { Dilt }\end{array}$ \\
\hline Mean HR & $70.9 \pm 5.5$ & $74.3 \pm 5.7$ & $71.0 \pm 6.3$ & 0.0712 & - & - & - \\
\hline $\begin{array}{l}\text { HR at time } \\
\text { of ischemia * } \\
\text { Total ischemia }\end{array}$ & $79.9 \pm 9.3$ & $82.7 \pm 10.7$ & $87.3 \pm 7.3$ & 0.6171 & - & - & - \\
\hline $\begin{array}{l}\text { Duration * } \\
\text { Ectopic atrial }\end{array}$ & $7.9 \pm 8.8$ & $2.5 \pm 3.8$ & $3.2 \pm 4.2$ & 0.0201 & $<0.05$ & $<0.05$ & $<0.05$ \\
\hline $\begin{array}{l}\text { beats * } \\
\text { Batimentos Atriais }\end{array}$ & $46.1 \pm 55.3$ & $19.2 \pm 31.9$ & $19.3 \pm 29.6$ & 0.0341 & $<0.05$ & $<0.05$ & NS \\
\hline $\begin{array}{l}\text { Ectopic } \\
\text { Batimentos } \\
\text { ventricular beats } *\end{array}$ & $\begin{array}{c}440 \pm 638 \\
5393 \pm 14664\end{array}$ & $\begin{array}{c}396 \pm 643 \\
3594 \pm 7408\end{array}$ & $3872 \pm 14070$ & 0.4493 & - & - & - \\
\hline
\end{tabular}

beats and ectopic ventricular beats when compared with placebo.
Effects on blood chemistry (Table V) - Serum glucose was not modified by any of the drug treatments. Serum po- 


\begin{tabular}{|lccccccc}
\hline \multicolumn{7}{c}{ Table V - Blood chemistry data } \\
\hline
\end{tabular}

tassium was reduced by chlorthalidone when compared with placebo and to diltiazem. However, chlorthalidone caused an increase in serum urea, serum triglycerides, and VLDL-cholesterol when compared with placebo and diltiazem. Diltiazem did not modify serum triglycerides and VLDL-cholesterol. Total cholesterol, HDL-cholesterol, and LDL-cholesterol were not modified by any of the treatments.

\section{Discussion}

Diuretics have been indicated as initial antihypertensive therapy ${ }^{7}$ because numerous randomized controlled trials have shown that they reduce cardiovascular morbidity and mortality ${ }^{8}$. Although some concern about the relationship between diuretic therapy and increased risk of death from coronary artery disease exists ${ }^{9}$, recent trials have shown a reduction of coronary heart disease in older hypertensive persons treated with these agents ${ }^{3-5}$. Our results are consistent with these trials and provide evidence that in elderly hypertensive patients with coronary artery disease, chlorthalidone has an antiischemic effect similar to the effect of diltiazem.

Few studies about the effects of diuretic therapy on myocardial ischemia exist. Nechwatal et al. ${ }^{10}$ reportimprovement in angina and an increase in exercise performance in patients with stable coronary artery disease treated with furosemide. More recently, Parker et al. ${ }^{6}$ reported that diuretic therapy improves exercise capacity in normotensive patients with stable angina. In our study, we found a borderline increase in exercise performance with the use of chlorthalidone.

Our study found that chlorthalidone reduced myocardial ischemia related to daily life activities in a homogeneous population of elderly hypertensive patients with myocardial ischemia and with normal left ventricular function. In fact, the antiischemic effect of diuretics may be even more significant in patients with ventricular dysfunction. In such patients, reduction in venous return plays an important role in reducing oxygen demand. The low dosage of chlorthalidone used in our study was associated with small and not cli- nically important metabolic effects (slight hypokalemia; mild increase in urea, triglycerides, and VLDL-cholesterol). This hypokalemia was not associated to with an increase in cardiac arrhythmias as found in the SHEP study ${ }^{11}$.

The mechanisms responsible for the antianginal effects of diltiazem have been well investigated and are related to a reduction in oxygen demand due to its negative inotropic and chronotropic effects and to improvement in oxygen supply by coronary vasodilatation and by an increased diastolic time ${ }^{12}$. On the other hand, the antiischemic effect of chlorthalidone is more difficult to explain. It is not related to chronotropic modification according to our observation on HR behavior on 48-hour ambulatory electrocardiogram monitoring. One possible explanation is the reduction in blood pressure. In fact, SBP and DBP in the office were reduced by chlorthalidone therapy, suggesting a blood pressure influence on antiischemic effects. Furthermore, Deedwania ${ }^{13}$ and Rehman ${ }^{14}$ found that an increase in SBP accompanies the onset of episodes of myocardial ischemia. However, our study did not measure blood pressure during daily life activities, so we had no evidence to support this hypothesis. Although the rate-pressure product did not change with the use of chlorthalidone, it is well known that the rate-pressure product is not the only determinant of oxygen demand because it does not consider changes in ventricular volume and contractility ${ }^{15}$. Another possible mechanism is a volemic reduction leading to decreases on venous return, left ventricular volume, left ventricular wall tension, and oxygen demand ${ }^{16}$. Nechwatal et al ${ }^{10}$ propose that the antianginal properties associated with the acute use of frusemide are due to a reduction in ventricular volumes and pressures resulting in decreased myocardial wall stress. Moreover, reduction of left ventricular end-diastolic pressure could lead to improvement in the coronary flow gradient leading to increased oxygen supply. A reduction in contractility has been suggested by Pierce ${ }^{17}$ to explain the antianginal effect of hydrochlorothiazide plus amiloride in patients with stable angina because amiloride was reported to have negative inotropic effects in animals. However, 
it is known that thiazide has no direct myocardial effect ${ }^{18}$ and so, this mechanism could not explain our results.

Study limitations - Because we included only elderly hypertensive patients with stable angina and without previous myocardial infarction, these results cannot be applicable to other subsets of patients. Furthermore, we studied a small population. Another limitation is that our study did not measure blood pressure during ambulatory electrocardiogram monitoring; therefore, we cannot exactly determine the role of blood pressure in decreasing myocardial ischemia during daily life activities.

Clinical implications - The present study shows that chlorthalidone therapy significantly reduces myocardial ischemia during daily life activities in hypertensive elderly patients with coronary artery disease. Our results are consistent with the SHEP study that showed 27\% fewer fatal and nonfatal myocardial infarctions in older patients with isolated systolic hypertension treated with low-dose chlorthalidone ${ }^{3}$ and a prevention of heart failure, especially in those with prior myocardial infarction ${ }^{19}$. Therefore, we can speculate that low-dose thiazide diuretic therapy may be beneficial and applicable for use in patients with refractory angina pectoris despite traditional treatment, especially in hypertensive subjects and in those with congestive heart failure.

Conclusion - In elderly hypertensive patients with coronary artery disease, chlorthalidone reduced myocardial ischemia similarly to diltiazem. This result is consistent with epidemiological studies and suggests that reduction of arterial blood pressure with thiazide therapy plays an important role in decreasing myocardial ischemia.

\section{Acknowledgment}

We are indebted to Mrs. Creuza Dal Bó for help with the statistical analysis.

\section{References}

1. MacMahon S, Peto R, Cutler J, et al. Blood pressure, stroke, and coronary heart disease. Part 1, prolonged differences in blood pressure: prospective observational studies corrected for the regression dilution bias. Lancet 1990; 335: 765-74.

2. National Center for Health Statistics. Vital statistics of the United States, 1988, vol. 2, mortality, part A. Washington, DC, Public Health Service, 1991.

3. SHEP Cooperative Research Group. Prevention of stroke by antihypertensive drug treatment in older persons with isolated systolic hypertension: final results of the Systolic Hypertension in the Elderly Program (SHEP). JAMA 1991; 265: 3255-64

4. MRC Working Party. Medical Research Council trial of treatment of hypertension in older adults: principal results. Br Med J 1992; 304: 405-12.

5. Dahlöf B, Lindholm LH, Hansson L, Scherstén B, Ekbom T, Wester PO. Morbidity and mortality in the Swedish Trial in Old Patients with Hypertension (STOPHypertension). Lancet 1991; 338: 1281-5.

6. Parker JD, Parker AB, Farrel B, Parker JO. Effects of diuretic therapy on the development of tolerance to nitroglycerin and exercise capacity in patients with chronic stable angina. Circulation 1996; 93: 691-6.

7. The Joint National Committee on Prevention, Detection, Evaluation, and Treatment of High Blood Pressure. The Sixth Report of the Joint National Committee on Prevention, Detection, Evaluation, and Treatment of High Blood Pressure. Arch Intern Med 1997; 157: 2413-46

8. Psaty BM, Smith NL, Siscovick DS, et al. Health outcomes associated with antihypertensive therapies used as first-line agents: a systematic review and metaanalysis. JAMA 1997; 277: 739-45.

9. Black HR. Metabolic considerations in the choice of therapy for the patient with hypertension. Am Heart J 1991; 121: 707-15.
10. Nechwatal W, König E, Isbary J, Greding H, Stauch M. Haemodynamic and electrocardiographic effects of frusemide during supine exercise in patients with angina pectoris. Br Heart J 1980; 44: 67-74.

11. Kostis JB, Lacy CR, Hall WD, et al. The effect of chlorthalidone on ventricular ectopic activity in patients with isolated systolic hypertension. The SHEP study group. Am J Cardiol 1994; 74: 464-7.

12. Nadazdin A, Davies GJ. Investigation of therapeutics mechanisms of atenolol and diltiazem in patients with variable-threshold angina. Am Heart J 1994; 127 : 312-17.

13. Deedwania PC, Nelson JR. Pathophysiology of silent myocardial ischemia during daily life - hemodynamic evaluation by simultaneous electrocardiography and blood pressure monitoring. Circulation 1990; 82: 1296-1304.

14. Rehman A, Zalos G, Andrews NP, Mulcahy D, Quyyumi AA. Blood pressure changes during transient myocardial ischemia: insights into mechanisms. J Am Coll Cardiol 1997; 30: 1249-55

15. Ardehali A, Ports TA. Myocardial oxygen supply and demand. Chest 1990; 98 699-705.

16. Parker JO, Case RB, Khaja F, Ledwich JR, Armstrong PW. The influence of changes in blood volume on angina pectoris: a study of the effect of phlebotomy. Circulation 1970; 41: 593-604.

17. Pierce GN, Cole WC, Liu K, et al. Modulation of cardiac performance by amiloride and selected derivatives of amiloride. J Pharmacol Exp Ther 1993; 265: 1280-91.

18. LohmollerG,Lohmoller R, PfefferMA,Pfeffer JM,FrohlichED. Mechanism of immediate hemodynamic effects of chlorothiazide. Am Heart J 1975; 89: 487-92.

19. Kostis JB, Davis BR, Cutler J, et al, for the SHEP Cooperative Research Group. Prevention of heart failure by antihypertensive drug treatment in older persons with isolated systolic hypertension. JAMA 1997; 278: 212-16. 